\title{
Mechanism of Platelets Stimulation by Non Thermal Plasma
}

\author{
Mohammed Ubaid Hussein, Enas Soleman Yousif \\ Department of Physiology and Medical Physics, College of Medicine, Anbar University, Anbar, Iraq
}

Email address:

mmphysics361@gmail.com (M. U. Hussein)

\section{To cite this article:}

Mohammed Ubaid Hussein, Enas Soleman Yousif. Mechanism of Platelets Stimulation by Non Thermal Plasma. International Journal of Chinese Medicine. Vol. 2, No. 1, 2018, pp. 1-5. doi: 10.11648/j.ijcm.20180201.11

Received: October 2, 2017; Accepted: November 23, 2017; Published: February 1, 2018

\begin{abstract}
This study includes the constructing of plasma needle (plasma torch) at atmospheric pressure The torch is produced by applying high voltage $17 \mathrm{kv}$ and frequency $17 \mathrm{kHz}$. A total of seventy 70 Iraqi patients seeking for a medical consultation in Al-Ramadi Teaching Hospital includes in this study. The plasma treatment is applied in vitro experiments regarding clotting time, Prothrombin time. Patients are from both sexes and different age groups between (20-45 year) are included in this study. The results show that the decreasing of distances lead to increase in torch. Therefore, the plasma ions induce a number of clotting processes and thus accelerates blood clotting. It can be concluded that when the doses of exposure increases the clotting time decreases. Reducing the distance plays an important role in the generation of plasma, which leads to reduce blood clotting time, accelerates blood clotting, and stop bleeding. The Prothrombin time decreases after treatment with little, which have a significant role in the acceleration of blood clotting, as the mechanical Prothrombin turning fibrinogen into fibrin, which provides for the conglomerate platelets.
\end{abstract}

Keywords: Plasma Needle, Clotting Time, Prothrombin Time

\section{Introduction}

Plasma physics is a branch of physics science which appears as important science in the last centuries because its interest by the matter of the universe. plasma forms are the most of the universe matter. This is because the plasma has unique properties and it can be generated in different laboratory methods. In other words, different plasmas with different properties can be obtained and then, can be used for wide range of applications.

According to its temperature, plasma can be classified to cold or hot plasma. The temperature of the cold one is approximately equal to the room temperature that is because, it is non- equilibrium plasma, i.e. its electrons have more large temperature greater than that of the neutral particles. For this reason, the cold plasma was used widely as a tool for different medical issues because it does not cause any thermal damage for the tissues. [1]

Non thermal atmospheric pressure plasmas have many medical application on living surface such as the sterilization of living tissues without damage, wound healing and induction of apoptosis in malignant tissue [2].

In hospital setting, sterilization of living animal or human tissues with minimal damage to these tissues are of great importance. Chemical sterilization is commonly employed, however, this technique cannot be used for the sterilization of open wounds, ulcers or burns due to the extent damage they cause to punctured tissues.

Non thermal atmospheric plasma especially when it is applied directly is an effective tool for the sterilization of living tissues, besides when applied on the living tissue it does not get damaged during plasma treatment [3].

Platelets, or thrombocytes are small, disk shaped clear cell fragments (i.e. cells that do not have nucleus), 2-3 $\mu \mathrm{m}$ in diameter, [4] which are derived from fragmentation of precursor megakaryocytes. The average lifespan of a platelet is normally just 5 to 9 days. Platelets are a natural source of growth factors. They circulate in the blood of mammals and are involved in hemostasis, leading to the formation of blood clots. Platelets release a multitude of growth factors including platelet-derived growth factor (PDGF), a potent chemotactic agent, and TGF beta, which stimulates the deposition of extracellular matrix. Both of these growth factors have been shown to play a significant role in the repair and regeneration of connective tissues. Other healing-associated growth factors produced by platelets include basic fibroblast growth factor, 
insulin-like growth factor 1, platelet-derived epidermal growth factor, and vascular endothelial growth factor. Local application of these factors in increased concentrations through Platelet-rich plasma (PRP) has been used as an adjunct to wound healing for several decades. [5]

\section{Theory}

Plasma needle is non thermal plasma designed with diameter $1 \mathrm{~mm}$ from interior, this needle constitutes cylindrical tube made from glass material with length $100 \mathrm{~mm}$ interior this glass tube, put other cylindrical tube made from iron material with external diameter $3 \mathrm{~mm}$, this tube connect to anode from high voltage power supply about $12 \mathrm{kV}$ peak to peak, applied power was lasting of electrical discharge which calculated from simultaneous values of voltage and current and applied frequency $20 \mathrm{kHz}$, it through pass argon gas where discharge between electrode and space through needle hole where plasma generation outside from hole [6]

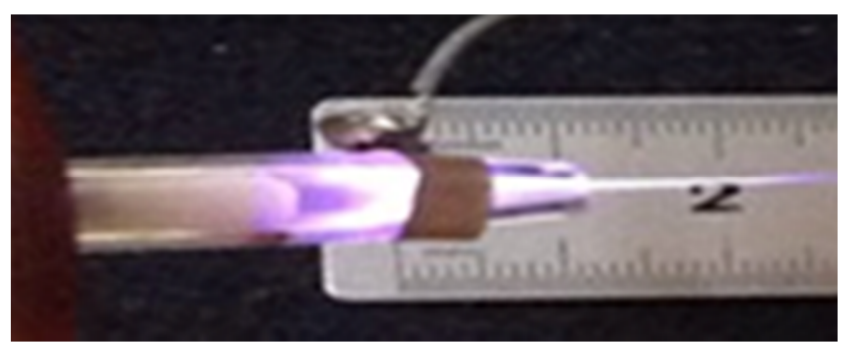

Figure 1. Non thermal plasma or plasma needle.

\section{Methods}

Seventy patients were complaining from different clinical signs and symptoms were admitted to Ramadi Teaching Hospital for seeking medical advise were included in the present study. No patient had previous operative or chemotherapeutic treatment, so the patients with hematological disorders (hemophilia) and those with open heart surgery that receiving warfarin and other antithrombotic agents were excluded from the study. The age range of these patients was (19-50), they were (45) male and (25) female.

\subsection{Coagulation Time}

It is the natural time required for blood to clot without the presence of any substance. The capillary tube method is used in the present study as:

The patient's finger was cleaned with spirit and was let to dry, the finger was pricked by lancet; the first drop of blood was removed, the finger was squeezed, to obtain a large drop of blood and the capillary tube was filled with blood, the capillary tubes were sealed plasticine and immersed in water bath at $37^{\circ} \mathrm{C}$, after one minute start breaking small pieces of the capillary tube every 30 second until a fibrin thread seen between the two broken ends [7, 8, 9]. From the same patient, another capillary tube was obtained exposed to plasma torch apparatus.

\subsection{Prothrombin Time}

It is most commonly measured using blood plasma. Blood is drawn into a test tube containing liquid sodium citrate, which acts as an anticoagulant by binding the calcium in a sample.

The blood is mixed, and then centrifuged to separate blood cells from plasma [10]. The same procedure was applied to another blood sample of the same patient, and then exposed to plasma needle apparatus.

The plasma is analyzed on an automated instrument at $37^{\circ} \mathrm{C}$ (as a nominal approximation of normal human body temperature), which takes a sample of the plasma. An excess of calcium is added (thereby reversing the effects of citrate), which enables the blood to clot again.

$0.2 \mathrm{ml}$ of sodium citrate is taken, put in (tube 1) and $1.8 \mathrm{ml}$ of blood is added, mixing and put in centrifuge for 3 minutes, and (tube 2) is taken and plasma is isolated from (tube 1) after centrifugation, put in incubator for 2 minutes with put can tube empty in incubator with temperature $37^{\circ} \mathrm{C}, 0.1 \mathrm{ml}$ of plasma is taken and put in can tube, $0.2 \mathrm{ml}$ of thromboplastin solution is added, then measure Prothrombin time before treatment with plasma torch $[11,1]$, same method is repeated after plasma torch exposure.

\section{Results}

The effects of plasma needle on Clotting Time CT and Prothrombin Time PT were shown. Seventy patients were complaining from different clinical signs and symptoms were admitted to Ramadi Teaching Hospital for seeking medical advise were included in the study. No patient had previous operative or chemotherapeutic treatment, so the patients with hematological disorders (hemophilia) and those with open heart surgery that receiving warfarin and other antithrombotic agents were excluded from the study. The age range of these patients was (19-50), they were $45(64.28 \%)$ males and 25 $(35.71 \%)$ females, as were shown in Figure 2.

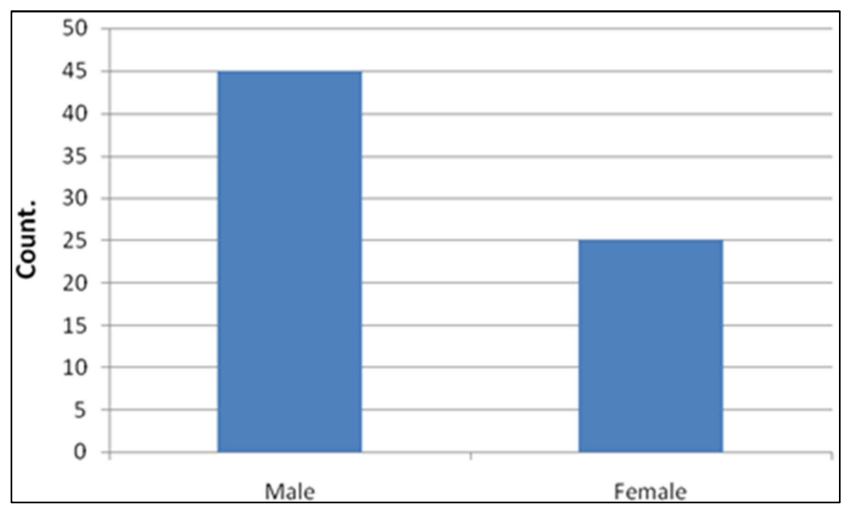

Figure 2. The sex distribution for the patients; males and females.

\subsection{The Clotting Time (CT)}

The blood clotting time is the time required for blood to clot in a glass tube. This time was measured for 70 patients before and after plasma needle treatments, and a comparison 
between the two cases were shown in Figure 3. The plasma was generated under the conditions of $17 \mathrm{kV}$ at frequency 17 $\mathrm{kHz}$ and $2 \mathrm{~cm}$ distance.
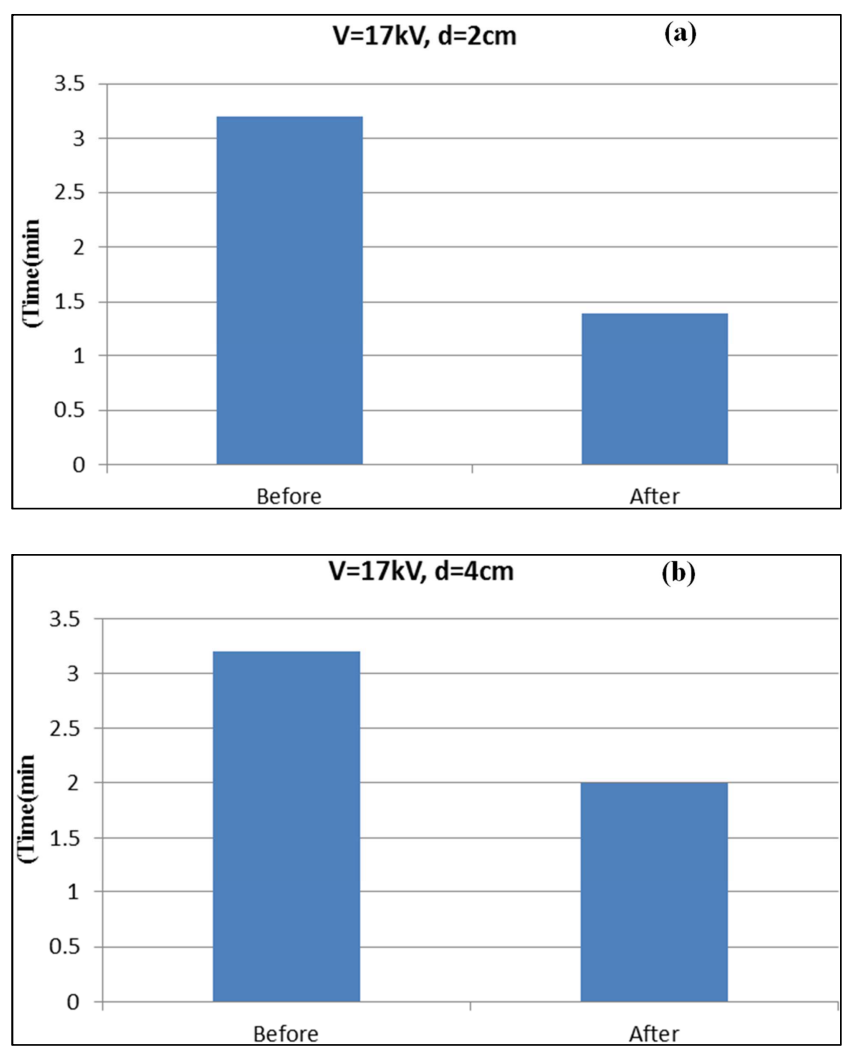

Figure 3. Mean values of blood clotting time before \& after cold plasma treatments when the distances are $(2 \mathrm{~cm}, 4 \mathrm{~cm})$ and applied voltage $17 \mathrm{kV}$.

The physical and medical affects of plasma needle significantly depend on the duration and distances as well as the applied voltages. it was affecting the homogeneity of the plasma and composition of biological activity of types generated plasma [12].

Then, to show the plasma treatment effect on the clotting time, when the distance becomes grater than the previous. The above measurements were repeated at distance $4 \mathrm{~cm}$. The results of this case were shown in Figure 3.

Figure 3. shows the mean values of clotting time of the studied patients before and after plasma treatment. It is clear that clotting time decreased with plasma treatment.

The reducing of the distances play an important role in the generation of plasma, where plasma characteristics in air depend on applied voltage as well as distances $[13,6]$. That was lead to reduce the blood clotting time and thus accelerates blood clotting.

When comparing for the same voltage, and variable distances. It is clear that the mean values of clotting time before and after treatment by plasma was different, this refers to the role of distance in the acceleration of blood clotting, so the increase in distance leads to the generation of plasma slim, thus decreasing the concentration of the ions, the acceleration does not occur in the blood clotting [1, 14].

As well as decreasing distance, the plasma ions induce a number of clotting processes, thus accelerates blood clotting and stop bleeding $[15,1]$.

The effective non-thermal plasma-medical system permits to achieve effective blood coagulation and stop bleeding without any thermal effects, ability of plasma to hasten wound healing through wound sterilization [15].

\subsection{Prothrombin Time (PT)}

Prothrombin time is rate at which prothrombin is converted to thrombin. This time was mesured for patients before and after plasma needle treatments. That was done under the conditions of $17 \mathrm{kV}$ applied voltage at frequency $17 \mathrm{kHz}$ and distances 2 and $4 \mathrm{~cm}$. A comparsion for its values before and after the plasma treatments was presented in Figure 4.

The mean values of Prothrombin time before treatment with plasma was 15.4 sec., while mean values of Prothrombin time after the treatment was $14 \mathrm{sec}$. in case (a). In case (b) the mean values of Prothrombin time before treatment with plasma was $15.4 \mathrm{sec}$., while mean values of Prothrombin time after the treatment was $14.8 \mathrm{sec}$. It means that, the plasma treatment decreases the Prothrombin time 1.4 sec. in case (a) while $0.6 \mathrm{sec}$. in case (b).
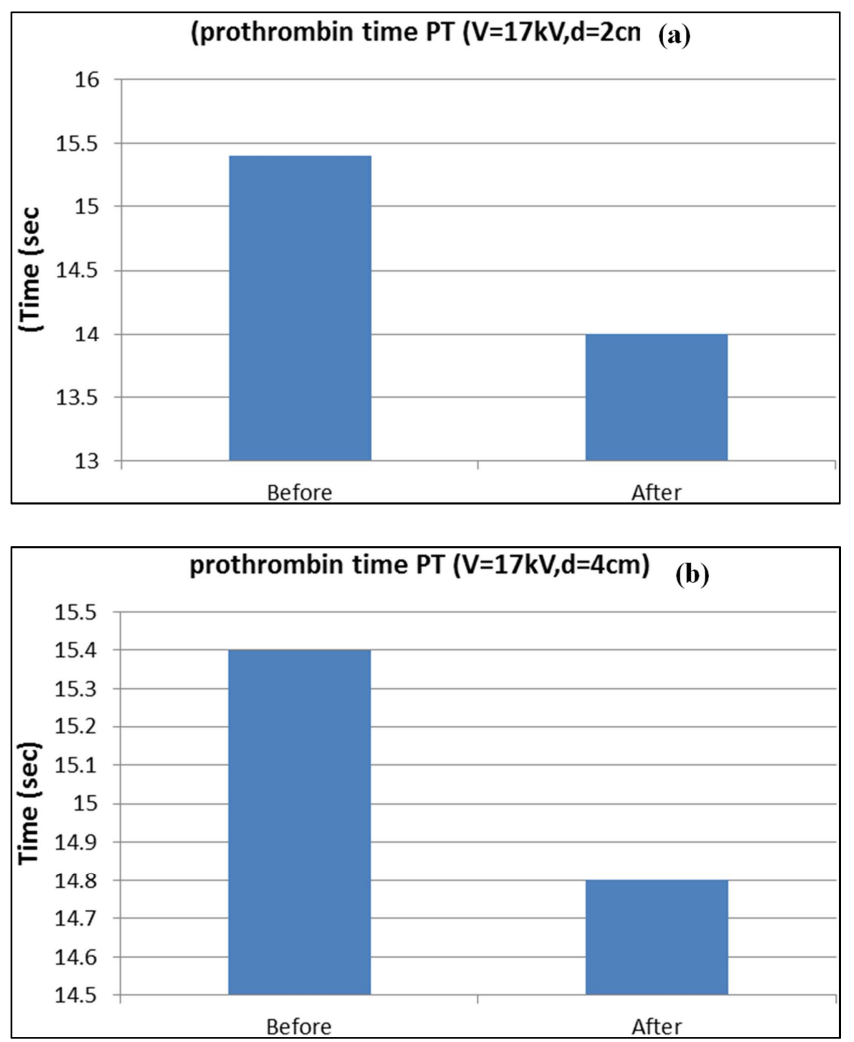

Figure 4. Mean values of Prothrombin time before \& after plasma treatments when applied voltage is $17 \mathrm{kV}$ at frequency $17 \mathrm{kHz}$ and the distances are a) $2 \mathrm{~cm}$ and b) $4 \mathrm{~cm}$.

The average Prothrombin time decreased after plasma treatment, which have a significant role in the acceleration of blood clotting, as the mechanical Prothrombin turning fibrinogen into fibrin, which provides for the conglomerate platelets [16]. 
That plasma is able to promote platelet activation and formation of fibrin filaments, platelets release a multitude of growth factors including platelet-derived growth factor (PDGF), a potent chemotactic agent, and Transforming Growth Factor- $\beta$ (TGF- $\beta$ ), which stimulates the deposition of extracellular matrix. Both of these growth factors have been shown to play a significant role in the repair and regeneration of connective tissues [17]. Other healing-associated growth factors produced by platelets include basic fibroblast growth factor, insulin-like growth factor 1, platelet-derived epidermal growth factor, and vascular endothelial growth factor (VEGF) $[18,1]$.

\section{Discussions}

The cold plasma and using plasma needle has the ability to accelerate blood clotting effect on the concentrations of blood proteins normal, so the ions existing in cold plasma stimulate number of clotting processes, and the greater the concentration of ions, this leads to an acceleration in the coagulation, hence conclude the plasma ion concentrations affects the blood and the likelihood of being able to stimulate blood clotting.

Regarding the previous test CT, PT, the use of plasma needle has a major role in hematological parameter this is due to catalyzes clotting factors (factor 8), platelet -derived growth factor (PDGF), Vascular endothelial growth factor (VEGF) and so forth.

Analysis of changes in concentration of various blood proteins and clotting factors indicates that plasma aids in promoting the advancement of blood coagulation, or in other words. Plasma is able to catalyze the complex biochemical processes taking place during blood coagulation $[19,1,6]$.

Direct non-thermal plasma can trigger natural, rather than thermally induced, coagulation processes; it was observed that the release of calcium ions and change of blood $\mathrm{PH}$ level, which could be responsible for coagulation, is insignificant $[20,1]$.

Plasma treatment is shown to "consume" coagulation factors (proteins and enzymes) and a visible film is formed on the surface of the treated samples. Increase in the sample volume and keeping the surface area fixed decrease the effect, indicating that plasma treatment initiates clot formation at the surface, not in the volume [1, 19, 20, 21].

\section{Conclusions}

Plasma needle was produced by applying high voltage $17 \mathrm{kv}$ and frequency $17 \mathrm{kHz}$. The results showed that the decreasing of distances lead to increases in torch and therefore, the plasma ions to induce a number of clotting processes and thus accelerates blood clotting. It can be concluded that when the doses of exposure increases the clotting time decreases. Reducing the distance play an important role in the generation of plasma, which leads to reduce blood clotting time and thus accelerates blood clotting and stop bleeding. The Prothrombin time decreases after treatment with little, which have a significant role in the acceleration of blood clotting.

\section{Acknowledgements}

We would like to express special words of thanks with deepest appreciation to Collage of Medicine and Clinical Laboratories in Al-Ramadi General Hospital, also the Staff working in these Laboratories.

\section{References}

[1] Mohammed U. Husain, "Low-Temperature Plasma Interactions in Medical Applications", Ph.D. Thesis, University of Baghdad, May, 2015.

[2] Ayan H., Fridman G., Gutsol A. F., Vasilets V. N., Fridman A. "Nanosecond-pulsed uniform dielectric-barrier discharge", IEEE Transactions on Plasma Science, 36, 504-508, 2008.

[3] Hoskinson A., Hershkowitz N. and Ashpis D., "Comparisons of force measurement methods for DBD plasma actuators in quiescent air," AIAA, 485, 2009.

[4] Campbell, Neil A. (2008). Biology (8th ed.). London: Pearson Education. p. 912. ISBN 978-0-321-53616-7. "Platelets are pinched-off cytoplasmic fragments of specialized bone marrow cells. They are about $2-3 \mu \mathrm{m}$ in diameter and have no nuclei. Platelets serve both structural and molecular functions in blood clotting".

[5] McAleer JP, Sharma S, Kaplan EM, Persich G (2006). "Use of autologous platelet concentrate in a nonhealing lower extremity wound". Adv Skin Wound Care 19 (7): 354-63.

[6] Mohammed U. Hussein," Plasma Needles on the Teeth Structure and Compressibility". The Arab Journal of Sciences \& Research Publishing, Vol. 2 - Issue (4): 2016, 5, 15 P. 4650; Article no: AJSRP/M15216.

[7] Mahapatra B. \& Mishra N., "Comparison of Bleeding Time and Clotting Time in Different Blood Groups", American Journal of Infectious Diseases 5, 2, 106-108, 2009.

[8] Ivy A. C., Nelson D., \& Beecher G., J. Lab. Clinical Medicine, 26, 1812, 1949.

[9] McKenzie, Shirlyn B., "Clinical Laboratory Hematology", 785-786, 1954.

[10] Fritsma, George A. "Evaluation of Hemostasis." Hematology: Clinical Principles and Applications. Ed. Bernadette Rodak. W. B. Saunders Company: Philadelphia, 719-53, 2002.

[11] Horsti J, Uppa H, Vilpo JA, "Poor agreement among prothrombin time international normalized ratio methods: comparison of seven commercial reagents," Clin. Chem,. 60$553,3,51,2001$.

[12] Fridman G., Shereshevsky A., Jost M. M., Brooks A. D." BioMedical Applications of Non-Thermal Atmospheric Pressure Plasma" in 37th AIAA Plasma dynamics and Lasers Conference. San Francisco, California., 2006.

[13] Kuchenbecker, M., Bibinov, N., kaemlimg, A., Wandke, D., Awakowicz, P. \& Viol, W., "characterization of DBD plasma source for biomedical applications". J. Phys. D: Appl. phys., 42, 045212, 2009. 
[14] Yildrim E. D., Ayan H., Vasilests V. N., Fridman G \& Sum, W. "Effect of dielectric barrier discharge plasma on the attachment and proliferation of osteobalasts cultured over poly (E-caprolacton) scaffolds",. Plasma process. Polym., 5, 58-66, 2008.

[15] Deng X T, Shi J, Chen H L and Kong MG, "Protein destruction by atmospheric pressure glow discharges", Appl. Phys. Lett., 90, 013903, 2007.

[16] Gregory Fridman, Alexey Shereshevsky, Marie Peddinghaus, Alexander Gutsol, Victor Vasilets, Ari Brooks, Manjula Balasubramanian, Gary Friedman, Alexander Fridman," BioMedical Applications of Non-Thermal Atmospheric Pressure Plasma" Plasma Medicine Lab, Drexel Plasma Institute, Drexel University, 3141 Chestnut, Philadelphia, PA 19104 2006.

[17] O'Connell SM, Impeduglia T, Hessler K, Wang XJ, Carroll RJ, Dardik H. "Autologous platelet-rich fibrin matrix as cell therapy in the healing of chronic lower-extremity ulcers". Wound Repair Regen, 16, 6, 749-56, 2008.
[18] Knighton DR, Ciresi KF, Fiegel VD, Austin LL, Butler EL "Classification and treatment of chronic nonhealing wounds. Successful treatment with autologous platelet-derived wound healing factors (PDWHF)". Ann. Surg., 204, 3, 322-30, 1986.

[19] Balasubramanian, M., "Blood conservation and coagulation by non-thermal dielectric barrier discharge technology." Transfusion, 47, 2, 6, 2007.

[20] Fridman G., Peddinghaus M., Balasubramanian M., H. Ayan, Fridman A., Gustol A., and Brooks A. "Blood Coagulation and living tissue sterilization by floating -electrode dielectric barrier discharge in air", plasma chem. plasma process., 26, 425-442, 2006.

[21] Mohammed, U. Hussein, Rana T. Mohsen, 2016. "Water pollution removal by Non- thermal plasma jet". Mesopotamia Environmental Journal, Mesop. environ. j., Spicial Issue A.; 86-92 (proceding of 6th International conference for Environmental Science-University of Babylon). 